\title{
Associations of Maternal Controlling Feeding Practices with Child Internalizing Symptoms and Body Mass Index in Ethnically-Diverse Mother-Child Dyads
}

\author{
Paulina Mozdzierz, Genevieve F. Dunton, \& Tyler B. Mason* \\ Department of Preventive Medicine, University of Southern California, Los Angeles, CA \\ https:/ / doi.org/10.33697/ ajur.2020.024 \\ Student:pmozdrie@usc.edu \\ Mentors:tylermas@usc.edu*,dunton@usc.edu
}

\begin{abstract}
Mothers may use controlling feeding practices (i.e., pressure to eat and restriction) to regulate their child's weight. However, these practices may have unintended consequences on the weight and mental health of children. The first aim of this study was to investigate differences in maternal controlling feeding practices by child gender, age, and maternal ethnicity. The second aim was to examine cross-sectional associations among maternal controlling feeding practices, child body mass index $z$-scores (BMI-z), global internalizing symptoms (i.e., depression and anxiety symptoms), and self-esteem. The third aim was to determine whether child sex and mother ethnicity moderate these associations. A sample of 202 ethnically diverse mother-child dyads (children ages 8-12; 49\% female) completed self-report questionnaires and had weight and height measurements taken. Results showed no differences in maternal controlling feeding practices by gender, ethnicity, or age. Pressure to eat was negatively related to child BMI-z, and restriction was positively related to BMI-z. Moreover, pressure to eat was negatively related to child self-esteem. There were no associations between maternal controlling feeding practices and global internalizing symptoms. Further, no associations differed by child gender or mother ethnicity. Maternal controlling feeding practices may be used to move a child's weight toward a healthy weight range. Overall, there was little evidence for associations between feeding practices and poor mental health; although, pressure to eat was related to poorer self-esteem in children.
\end{abstract}

\section{KEYWORDS}

Maternal; Feeding; Practices; Child; BMI-z; Mental; Health; Controlling; Restricting

\section{INTRODUCTION}

Obesity is increasingly becoming a concern in children of all ages, genders, and ethnicities. ${ }^{1,2}$ Similar to statistics for obesity, approximately one in every five children and adolescents have a mental health problem. ${ }^{3-6}$ Higher body weight and mental health problems, both of which have been found to correlate with controlling feeding practices, can lead to numerous health complications that may persist well into adulthood. ${ }^{5,6,7}$ Further, studies have found associations between higher body weight in childhood and an increased prevalence of anxiety and mood disorders later in life. ${ }^{8}$ Particularly, middle childhood is a time where mental health disorders and weight gain begin developing. ${ }^{6, ~ 8, ~ 11-13 ~}$

While obesity itself may lead to negative consequences such as depression, anxiety disorders, and chronic disease risk, ${ }^{8,14-16}$ it is possible that efforts used to control children's weight, such as controlling feeding practices, may contribute to these factors and pose an even greater risk for these physical and mental health problems. ${ }^{8}$ Previous studies have found that controlling feeding practices can contribute to a failure to appropriately respond to hunger or fullness signals, which can lead to weight gain. Research suggests that children tend to overeat when given free access to foods that are typically restricted from their diet.17,18 Further, feeding practices may lead to the development of weight and eating behavior concerns and a lack of autonomy. ${ }^{19}$ Additional research in adolescents found that maternal controlling feeding practices were positively correlated with bulimic symptoms (in females) and negatively correlated with self-esteem. ${ }^{6,20-21}$

Given healthcare providers' focus on the importance of proper nutrition and maintenance of a healthy weight in children,,10 parents may use controlling feeding practices (i.e., restricting child's food intake and pressuring a child to eat) in order to help their child achieve and maintain a healthy weight, which is defined by body mass index (BMI) for age and sex from the 5th through the 85th percentile. ${ }^{11}$ Parents may restrict eating by limiting meal portions and commenting on the large intake of food by their child, 
or parents may pressure their child to eat by forcing their child to consume an entire meal or punishing their child for failure to finish a meal. ${ }^{12,20,22-24}$ Among infants and toddlers, controlling feeding practices, both restriction and pressure to eat, have been shown to predict lower weight in children. ${ }^{11-12}$ Despite being predictive of lower weight in infants, restrictive and controlling feeding practices may be associated with poor weight and mental health-related outcomes in older children., 12-13

Research findings have not been consistent regarding the relationship between parental controlling feeding practices and child weight status. Some research has found positive associations between restrictive feeding practices and weight and negative associations between pressure to eat and weight in early childhood.13,25-26 This finding could be due to child overeating behavior when given the opportunity to eat away from the supervision of a restrictive parent. ${ }^{27}$ Pressure to eat may reduce weight due to the development of aversion or anxiety towards food; in the absence of parental pressure, the child refuses to eat. ${ }^{27-28}$ In contrast, other studies of toddlers found no relation between child BMI-z scores and maternal controlling feeding practices; ${ }^{27}$ Similarly, in separate studies of Hispanic preschool children, there was no relation between maternal controlling feeding practices and BMI-z scores. ${ }^{23,29}$

Empirical research has primarily focused on how maternal feeding practices can affect the physical weight of a child. Thus, there is a lack of data on the relationship between maternal feeding practices and child internalizing symptoms and self-esteem. One study found a positive relationship between increased maternal internalizing symptoms and increased controlling feeding practices ${ }^{30}$ but studies have yet to look at child internalizing symptoms. Studies have found positive correlations between restrictive maternal feeding practices and disordered eating, such as binge eating in adolescents. ${ }^{20,24}$ Although disordered eating is highly associated with internalizing symptoms, ${ }^{31}$ it is unclear if the link between maternal controlling feeding practices extends to internalizing symptoms, such as mood and anxiety symptoms.

The Role of Child Gender

Gender may be an important variable to consider when examining the associations between maternal feeding practices and child internalizing symptoms and weight. Findings on gender and maternal feeding practices are varied; some studies suggest that maternal restriction is associated with self-esteem in girls with obesity and also associated with food intake control in girls, yet no significant associations were found for boys. ${ }^{32}$ This finding suggests that girls may be disproportionately affected by controlling feeding practices. Additional research observed that maternal pressure to eat in children 7-12 years old was linked to greater consumption of food in the absence of hunger, and a stronger correlation was observed for boys compared to girls. ${ }^{21.33-34}$ One study found that fathers reported more pressure to eat for boys than girls. ${ }^{35}$ However, in a separate study comparing maternal and paternal feeding practices among parents of boys and girls, there were no differences. ${ }^{20}$ Additional research found that maternal feeding is more likely to occur between mother-daughter than mother-son relationships; one possible explanation for this difference is the societal pressure to adhere to gender-related dietary norms. ${ }^{36-38}$ These associations provide preliminary evidence of gender being a possible variable to consider when assessing maternal controlling feeding practices and their relation to child weight and internalizing symptoms.

\section{The Role of Ethnicity}

In addition to gender, ethnicity may be an important factor to consider. Lifestyle differences between Hispanic and non-Hispanic families may lead to behavior that results in varied child weight and internalizing symptoms. Findings are mixed. However, some evidence suggests that non-White Hispanic mothers restrict foods less than White mothers. Other studies did not find evidence to support this; some research has found that Hispanic mothers were more likely to pressure their children to eat. ${ }^{38-39}$ One study found that non-White parents, including Hispanic parents, engaged in more restrictive and pressuring feeding practices. ${ }^{40}$

Differences between Hispanic and non-Hispanic mothers may be linked to Hispanic culture, which emphasizes social eating. ${ }^{41-43}$ In Hispanic culture, the ideal maternal figure is closely associated with feeding children; the more food, the more nurturing a mother is. ${ }^{44}$ This can lead to maternal pressuring to eat. Research suggests that Hispanic mothers have a different idea of what being overweight is, and thus they participate in pressuring to eat at times when non-Hispanic parents would not. ${ }^{45-46}$ Studies indicate that non-White Hispanic mothers may prefer their children to be heavier. They believe that this indicates child health and strength; this mentality can lead to more pressuring and less restrictive feeding practices. ${ }^{41,45-47}$

\section{The Current Study}

Varying patterns of feeding practices by gender and ethnicity have led us to expect differences between Hispanic and nonHispanic children in relation to how maternal feeding patterns affect child internalizing symptoms and weight. We expect these differences due to cultural differences; for example, Hispanics regard food as a core part of their culture and thus are less likely to restrict it. ${ }^{19}$ Gender differences are expected due to the societal norms placed on females and males, such as that females should eat less than males, as well as the difference in mother-daughter and mother-son relationships. ${ }^{36-37,48}$ These patterns could explain 
differences in child obesity and internalizing symptoms, thus establishing the importance of the further study of these associations. ${ }^{15}$

Studying the effect of maternal controlling feeding practices on adolescents, ages $8-12$, is important because adolescence is a time where children strive to be more independent; they make their own decisions, yet decisions are still influenced by decisions made for them growing up. Additionally, mental health disorders are emerging in this age group, and it is important to study the role of controlling feeding practices in this trend. $6,8,11-13$

The current study had several goals. The first research question examined differences in maternal controlling feeding practices by child gender, age, and maternal ethnicity. Age was examined as children become more independent as they get older, and mothers' controlling feeding practices may change due to increased autonomy. ${ }^{49}$ The second research question was examined to see if bivariate associations were among maternal feeding practices, child BMI, child internalizing symptoms (i.e., depressive and anxiety symptoms), and self-esteem. The third research question examined child gender and maternal ethnicity as moderators of associations between maternal controlling feeding practices and child BMI, internalizing symptoms, and self-esteem. The study aims to add knowledge to a topic that has not been extensively studied. Specifically, findings will allow us to understand the effect of controlling feeding practices on children's mental health and how this may differ for children of different gender and ethnicity. Findings may be used to develop interventions to educate parents on controlling eating habits that best promote healthy child weight and mental health, thus bettering health outcomes during adolescence and into adulthood.

\section{METHODS AND PROCEDURES}

\section{Participants and Procedure}

The current study sample was drawn from Wave one of the Mothers, and Their Children's Health (MATCH) study, which includes 202 dyads of ethnically diverse mothers and their 8-12-year-old children (age range during baseline assessment). The sample included 103 boys and 99 girls, and the mean age of children was 9.60 (SD=0.91). There were 99 mothers (49\% of dyads) who indicated being Hispanic or Latina, with 103 being non-Hispanic. Mother-child dyads were recruited from urban schools in the greater Los Angeles community. Children were in third through sixth grade. Inclusion criteria included $\geq 50 \%$ custodianship of the child with the mother and ability of mothers and children to read in either English or Spanish. The study exclusion criteria for children or mothers: (1) currently taking medications for thyroid function or psychological conditions such as depression, anxiety, mood disorders, and ADHD, (2) health issues that limit physical activity, (3) enrolled in special education programs, (4) currently using oral or inhalant corticosteroids, (5) pregnancy, (6) mother works more than two weekday evenings per week (e.g., between 5 and $9 \mathrm{pm}$ ) or more than eight hours on any weekend day, and (7) child classified as underweight by a BMI percentile $<5 \%$ adjusted for age and sex.

The study was reviewed and approved by the institutional review board at the University of Southern California and Northeastern University. Dyads were recruited. Mothers provided consent for their own participation and parental consent for their child. Children provided assent. After this process, dyads completed paper and pencil questionnaire measures. A trained research assistant took weight and height measures. Dyads were given additional paper and pencil measures to take home to complete and return to the researchers. Mothers and children each received $\$ 100$ compensation for their time and effort in the first wave of the study.

\section{Measures}

Child BMI. Height and weight were measured using an electronically calibrated digital scale and professional stadiometer. These measures were used to calculate BMI $\left(\mathrm{kg} / \mathrm{m}^{2}\right)$ for mothers and age- and gender-specific body mass index percentiles for children.

Child internalizing symptoms. The Revised Children's Anxiety and Depression Scale (RCADS) ${ }^{50}$ was used to assess child internalizing symptoms. The following subscales were measured: generalized anxiety, major depression, panic disorder, and separation anxiety. Some sample items include: "I feel sad or empty" and "I worry that bad things will happen to me." This 47-item questionnaire utilizes a response scale ranging from 0 (never) to 3 (always) for each item. A total score was calculated with higher scores indicating greater internalizing symptoms. A systematic review and meta-analysis showed that the RCADS has adequate psychometric properties across various assessment settings, languages, and locations. ${ }^{51}$

Child self-esteem. The Rosenberg Self-Esteem (RSE) ${ }^{52}$ scale was used to assess child global self-esteem. This 10 -item scale asks participants to reflect on their feelings about themselves on a scale ranging from 1 (strongly disagree) to 4 (strongly agree). Sample items include: "I feel that I have a number of good qualities" and "I am able to do things as well as most other people." The RSE has shown good convergent validity for students in high school and college students, men, women, various ethnic groups, and reliability and concurrent validity for high school students. ${ }^{53-54}$ 
Maternal controlling feeding practices. The Child Feeding Questionnaire (CFQ) ${ }^{55}$ utilizes self-reported measures to determine parental beliefs, attitudes, and practices and their effects on child feeding. Restrictive \& Pressure to Eat subscales were used in the current study. The eight-item Restriction Eating subscale assessed restrictive pressures enforced by parents on the type and amount of food a child eats. A sample item is, "I have to be sure that my child does not eat too many high-fat foods." The eight-item Pressure to Eat subscale assesses the frequency of pressuring the child to eat more food. A sample item is, "My child should always eat all of the food on her plate." Mothers indicated on a scale from 1 (disagree) to 5 (agree) as to how much they engaged in various feeding behaviors. Studies found good psychometric properties of the CFQ in Hispanic and non-Hispanic mothers. ${ }^{29,55-56}$

\section{Statistical Analyses}

Analysis of covariance (ANCOVA) models were calculated to examine main effects and two- and three-way interactions among child gender, child age, and mother ethnicity in relation to maternal feeding practices (i.e., restrictive eating and pressure to eat). Age was used as a covariate given developmental influences on maternal feeding practices. ${ }^{49}$ To answer the second research question, bivariate Pearson correlations and descriptive statistics were calculated among maternal feeding practices and BMI-z, global internalizing symptoms, and self-esteem. Three hierarchical linear regression analyses were used to examine differences in the effect of maternal feeding practices on internalizing symptoms, self-esteem, and BMI-z, moderated by gender and ethnicity. Two-way interaction terms were created between gender and maternal feeding practices and ethnicity and maternal feeding practices. Age was included as a covariate in regression analyses. In the hierarchical linear regression, the first step included age; the second step included age, gender, ethnicity, and maternal feeding practice main effects; the third step added the interactions between gender and maternal feeding practices and ethnicity and maternal feeding practices. Significance tests were conducted at $p<.05$ level.

\section{RESULTS}

\section{Research Question 1}

There were no significant two- or three-way interactions among child age, child gender, and mother ethnicity in relation to pressure to eat or restriction. Thus, interactions were removed from models. Neither child gender $(p=.96)$ nor mother ethnicity $(p=.52)$ were related to pressure to eat. Older children had mothers who reported greater pressure to eat $(p=.01)$. Neither child gender $(p=.17)$ nor mother ethnicity $(p=.38)$ were related to restriction. Older children had mothers who reported greater restriction $(p=.04)$

\section{Research Question 2}

Bivariate correlations are presented in Table 1. Pressure to eat and restriction were only weakly positively correlated. Pressure to eat was significantly related to lower BMI-z scores and lower child self-esteem scores. Greater restriction was significantly, yet weakly, associated with higher BMI-z scores. There were no associations between maternal controlling feeding practices and global internalizing symptoms.

\begin{tabular}{lllllc}
\hline & $\mathbf{1}$ & $\mathbf{2}$ & $\mathbf{3}$ & $\mathbf{4}$ & $\mathbf{5}$ \\
\hline 1. Maternal pressure to eat & - & $.16^{*}$ & $-.41^{* * *}$ & .07 & $-.23^{* *}$ \\
2. Maternal restriction & & - & $.20^{* *}$ & .09 & -.04 \\
3. Child BMI-z & & & - & .12 & -.09 \\
4. Child internalizing symptoms & & & & - & $-.59^{* * *}$ \\
5. Child self-esteem & & & & & - \\
\hline $\boldsymbol{M}$ & 2.39 & 3.03 & 0.52 & 0.58 & 31.88 \\
$\boldsymbol{S D}$ & 1.07 & 0.97 & 1.05 & 1.05 & 5.31 \\
Minimum & 1.00 & 1.00 & -2.63 & 0.00 & 13.00 \\
Maximum & 5.00 & 5.00 & 2.61 & 2.23 & 40.00 \\
Skewness & 0.36 & -0.24 & -0.16 & 1.36 & -0.78 \\
Kurtosis & -0.95 & -0.73 & -0.51 & 2.42 & 0.64 \\
\hline
\end{tabular}

Note. BMI=body mass index. ${ }^{* * *} \mathrm{p}<.001,{ }^{*} * p<.01,{ }^{*} p<.05$

Table 1. Descriptive Statistics among Study Variables. 
Research Question 3

Results of hierarchical linear regression analyses are displayed in Table 2. Older children had fewer internalizing symptoms and better self-esteem across steps. There were no significant statistical interactions among maternal controlling feeding practices and gender or ethnicity in relation to any dependent variables. Further, results showed similar patterns on findings as bivariate correlations.

\begin{tabular}{|c|c|c|c|c|c|c|c|c|c|}
\hline & \multicolumn{3}{|c|}{ BMI-z } & \multicolumn{3}{|c|}{ Internalizing Symptoms } & \multicolumn{3}{|c|}{ Self-Esteem } \\
\hline & $B$ & $\mathrm{SE}$ & $p$ & $B$ & $\mathrm{SE}$ & $p$ & $B$ & SE & $p$ \\
\hline \multicolumn{10}{|l|}{ Step 1} \\
\hline Child Age & .09 & .08 & .26 & -.76 & .25 & .003 & 1.31 & .44 & .003 \\
\hline \multicolumn{10}{|l|}{ Step 2} \\
\hline Child Age & .02 & .07 & .82 & -.75 & .26 & .01 & 1.00 & .44 & .02 \\
\hline Pressure to eat & -.46 & .06 & $<.001$ & .16 & .23 & .47 & -1.12 & .37 & .003 \\
\hline Restriction & .31 & .07 & $<.001$ & .18 & .25 & .47 & -.10 & .41 & .81 \\
\hline Female & .15 & .13 & .25 & .10 & .47 & .83 & -1.50 & .77 & .05 \\
\hline Hispanic & .34 & .13 & .01 & .82 & .47 & .08 & -.83 & .77 & .28 \\
\hline \multicolumn{10}{|l|}{ Step 3} \\
\hline Child Age & .01 & .07 & .93 & -.76 & .27 & .01 & .99 & .45 & .03 \\
\hline Pressure to eat & -.45 & .11 & $<.001$ & -.26 & .38 & .49 & -.68 & .64 & .29 \\
\hline Restriction & .33 & .12 & .01 & .31 & .42 & .45 & .32 & .71 & .66 \\
\hline Female & .13 & .13 & .31 & .10 & .47 & .83 & -1.47 & .78 & .06 \\
\hline Hispanic & .37 & .13 & .01 & .90 & .47 & .06 & -.91 & .78 & .25 \\
\hline Pressure to eatXFemale & .20 & .13 & .12 & .60 & .45 & .19 & -.70 & .74 & .35 \\
\hline Pressure to eatXHispanic & -.25 & .13 & .05 & .20 & .45 & .66 & -.09 & .74 & .90 \\
\hline RestrictionXFemale & -.11 & .14 & .45 & -.57 & .50 & .26 & .28 & .83 & .74 \\
\hline RestrictionXHispanic & .11 & .14 & .46 & .34 & .50 & .49 & -1.10 & .83 & .19 \\
\hline
\end{tabular}

Table 2. Hierarchical Linear Regressions of Outcomes on Maternal Controlling Feeding Practices, Gender, Ethnicity, and Interactions.

\section{DISCUSSION}

This cross-sectional study examined how maternal controlling feeding practices were associated with weight, global internalizing symptoms, and self-esteem among 8-12-year-old children and how child gender and mother ethnicity may moderate these relationships. There were no differences in reported maternal controlling feeding practices by child gender or mother ethnicity, but older children had mothers who reported greater restriction. This pattern of findings suggests that other factors may be more salient as to which mothers are more likely to use these practices with children of this age range, such as maternal mental health and parenting styles. ${ }^{30,57}$ 
Maternal pressure to eat was associated with lower child BMI z-scores, and maternal restriction was associated with higher child BMI-z scores. Mothers may be more likely to pressure lower weight children to eat more food as they may perceive their child as not getting appropriate nutrition. However, of note, severely underweight children were excluded. Oppositely, mothers may restrict the food intake of higher weight children given the health detriments and social stigma associated with being overweight and obese. Some prior research has shown no associations between maternal controlling feeding practices and BMI among Hispanics ${ }^{23-29}$; however, the current results found that ethnicity did not moderate the significant associations. Given the increasing public health focus on maintaining healthy child weight, ${ }^{43}$ mothers of children, regardless of ethnicity, may become more focused on using controlling feeding practices with children outside of prescribed weight ranges.

Results indicated that pressure to eat and self-esteem scores were negatively correlated. One study showed that higher parental pressure to achieve was related to lower child self-esteem suggesting that undue parental pressure could potentially decrease child self-esteem..$^{59}$ Results of the current study show that this may extend to pressure to eat. Perhaps maternal pressuring reduces a child's feelings of autonomy, which then decreases their self-esteem and confidence to make decisions. ${ }^{60}$

There were no significant relationships among maternal controlling feeding practices and global internalizing symptoms. This is the first study to our knowledge that has examined this relation; however, studies have found associations between maternal controlling feeding practices and child bulimic symptoms and emotional eating scores. ${ }^{20,24} \mathrm{It}$ is possible that controlling feeding practices are limited to being associated with eating psychopathology and no other forms of psychopathology. Furthermore, maternal controlling feeding practices may be more detrimental to older adolescents' mental health or only in children at risk for eating disorders. Given the dearth of literature in this area, it is important to conduct more research on these relationships in diverse samples of mother-child dyads.

This study did not find any differences in the association of controlling feeding practices and weight and internalizing symptoms by gender and ethnicity. It is possible that differences between boys and girls may emerge later into adolescence. Continuous exposure to controlling feeding patterns at ages 13-15 has been correlated with unhealthy eating attitudes, such as restriction (more prevalent in boys as compared to girls) and pressuring oneself to eat (more prevalent in girls as compared to boys) ${ }^{61-62}$ Girls are more likely to diet during adolescence, as compared to boys; a past review indicated that 41-66\% adolescent girls diet, and 20$31 \%$ adolescent boys diet, both for the purpose of weight loss. Girls are more likely to diet because of society's perception of the ideal, extremely thin female body. Popular adolescent dieting methods, especially for girls, include crash diets, fasting, slimming tablets, diuretics, and laxatives. Peer pressure, media pressure, and perception of the harmlessness of dieting strategies perpetuate these behaviors. ${ }^{63-64}$ Internalized symptoms starting at a young age become the norm for these adolescents and thus follow them into adulthood. Further, ethnic differences may be more likely to emerge in Hispanic families who are less acculturated to Western society. ${ }^{44}$ More research is needed in adolescents and diverse groups of Hispanic dyads with regard to acculturation.

A limitation of the current study includes a cross-sectional analytic strategy, which limits the ability to infer causation and directionality of effects. This was a community-based sample that was generally well-adjusted and had fewer mental health problems compared to clinical samples. Samples with greater numbers of children with mental health problems may produce differing results. Thus, future studies will be improved by sampling from clinical populations, and future studies should consider examining differences between overt (i.e., controlling a child's food intake in a way that can be detected by the child such as verbally restricting or encouraging eating) versus covert (i.e., controlling a child's food intake in a way that cannot be detected by the child such as placing smaller portions on plates or reducing amounts of snack food available in-home) forms of pressure to eat and restricting, which differ in whether the child can detect the controlling behavior. ${ }^{34,65}$ Overt and covert control can be measured with a 9-item questionnaire that asks about direct and indirect controlling feeding practices. ${ }^{65}$ These constructs are difficult to measure, and further research needs to be done to improve current measures. Future studies may be improved by utilizing more accurate video observational methods, as compared to self-reporting of intake; this may aid in the standardization of measures ${ }^{66}$ Finally, parental stress, home environment, socioeconomic standing, single versus dual-parent households, food scarcity, and cultural versus ethnic differences should be considered in future studies as possible moderators or mediators.

While future studies are necessary to explore the relationships between maternal controlling feeding practices and child weight and internalizing problems, this study provided new insights into these associations and showed that associations are similar across gender and ethnicity.

\section{CONCLUSIONS}

Maternal pressure to eat was correlated with lower child BMI z-scores, and maternal restriction was correlated with higher child BMI-z scores. In general, there were no significant correlations between maternal controlling feeding practices and internalizing symptoms, as well as no significant differences based on gender and ethnicity of a child. However, more maternal pressure to eat was related to lower self-esteem. This is the first study, to our knowledge, that studied the effect of controlling feeding practices 
on global internalizing symptoms and self-esteem. Results provide some evidence that controlling feeding practices may be associated with less self-esteem in middle childhood, but there were no differences in relation to internalizing symptoms. More research will be needed to determine if controlling feeding practices impact mental health into adolescence. Also, results were similar across gender and ethnicity, suggesting that controlling feeding practices do not have a differential impact on mental health and weight in middle childhood. Future studies must be done to further explore the relationship between maternal feeding practices, child weight, and mental health, as well as the effect of gender and ethnicity, particularly in older children and samples with more variability in internalizing symptoms.

\section{ACKNOWLEDGEMENTS}

The author thanks everyone at the USC REACH lab for being so welcoming and for providing advice and suggestions throughout the duration of this project.

\section{REFERENCES}

1. Hruby, A., and Hu, F. B. (2015) The epidemiology of obesity: A big picture, Pharmacoeconomics 33, 673-689. doi:10.1007/s40273 014-0243-x

2. Ludwig, D. S. (2018) Epidemic childhood obesity: Not yet the end of the beginning, Pediatrics 141, e20174078. doi:10.1542/peds.2017-4078

3. Bor, W., Dean, A. J., Najman, J., and Hayatbakhsh, R. (2014) Are child and adolescent mental health problems increasing in the 21st century? A systematic review, Aust N Z J Psychiatry, 48, 606-616. doi:10.1177/0004867414533834

4. Fink, E., Patalay, P., Sharpe, H., Holley, S., Deighton, J., and Wolpert, M. (2015) Mental health difficulties in early adolescence: A comparison of two cross-sectional studies in England from 2009 to 2014, J Adolesc Health 56, 502-507. doi:10.1016/j.jadohealth.2015.01.023

5. Patel, V., Flisher, A. J., Hetrick, S., and Mcgorry, P. (2007) Mental health of young people: A global public-health challenge, Lancet 369, 1302-1313. doi:10.1016/s0140-6736(07)60368-7

6. Bennett, A. C., Brewer, K. C., and Rankin, K. M. (2011) The association of child mental health conditions and parent mental health status among U.S. children, 2007, Maternal Child Health J 16, 1266-1275. doi:10.1007/s10995-011-0888-4

7. Moussavi, S., Chatterji, S., Verdes, E., Tandon, A., Patel, V., and Ustun, B. (2007) Depression, chronic diseases, and decrements in health: Results from the World Health Surveys, Lancet 370, 851-858. doi:10.1016/s0140-6736(07)61415-9

8. Russell-Mayhew, S., McVey, G., Bardick, A., and Ireland, A. (2012) Mental health, wellness, and childhood overweight/obesity, J Obes 2012, 281801. doi:10.1155/2012/281801

9. Canadian Task Force on Preventive Health Care. (2015) Recommendations for growth monitoring, and prevention and management of overweight and obesity in children and youth in primary care, CMAJ 187, 411-421. doi:10.1503/cmaj.141285

10. Payne, L. O., Galloway, A. T., and Webb, R. M. (2011) Parental use of differential restrictive feeding practices with siblings, Int J Pediatr Obes 6, e540-546. doi:10.3109/17477166.2011.575144

11. Farrow, C. V., and Blissett, J. (2008) Controlling feeding practices: Cause or consequence of early child weight?, Pediatrics 121 , e164-e169. doi:10.1542/peds.2006-3437

12. Rodgers, R. F., Paxton, S. J., Massey, R., Campbell, K. J., Wertheim, E. H., Skouteris, H., and Gibbons, K. (2013) Maternal feeding practices predict weight gain and obesogenic eating behaviors in young children: A prospective study, Int J Behav Nutr Phys Act 10, 24. doi:10.1186/1479-5868-10-24

13. Boswell, N., Byrne, R., and Davies, P. S. W. (2018) An examination of children's eating behaviours as mediators of the relationship between parents' feeding practices and early childhood BMIz, Obes Sci Pract. doi:10.1002/osp4.320

14. Pi-Sunyer, F. X. (2002) The Medical Risks of Obesity. Obesity Surgery, 12(S1) doi:10.1007/bf03342140

15. Kearns, K., Dee, A., Fitzgerald, A. P., Doherty, E., \& Perry, I. J. (2014) Chronic disease burden associated with overweight and obesity in Ireland: The effects of a small BMI reduction at population level. BMC Public Health,14(1) doi:10.1186/14712458-14-143

16. Grucza, R. A., Krueger, R. F., Racette, S. B., Norberg, K. E., Hipp, P. R., \& Bierut, L. J. (2010) The Emerging Link Between Alcoholism Risk and Obesity in the United States. Archives of General Psychiatry, 67(12), 1301. doi:10.1001/archgenpsychiatry.2010.155

17. M.S. Faith, K.S. Scanlon, L.L. Birch, L.A. Francis, B. SherryParent-child feeding strategies and their relationships to child eating and weight status Obesity Research, 12 (11) (2004), pp. 1711-1722, doi: 10.1038/oby.2004.212

18. L.L. Birch, J.O. Fisher, K.K. Davison Learning to overeat: Maternal use of restrictive feeding practices promotes girls' eating in the absence of hunger American Journal of Clinical Nutrition, 78 (2) (2003), pp. 215-220, 10.1038/ $\mathrm{nrm} 2621$

19. Smokowski, P.R., Bacallao, M.L., Cotter, K.L. et al. Child Psychiatry Hum Dev (2015) 46: 333. https:// doiorg.libproxy1.usc.edu/10.1007/s10578-014-0474-2

20. Blissett, J., Meyer, C., and Haycraft, E. (2006) Maternal and paternal controlling feeding practices with male and female children, Appetite 47, 212-219. doi:10.1016/j.appet.2006.04.002 
21. Van Strien, T., and Bazelier, F. G. (2007) Perceived parental control of food intake is related to external, restrained and emotional eating in 7-12-year-old boys and girls, Appetite 49, 618-625. doi:10.1016/j.appet.2007.03.227

22. Haycraft, E. L., and Blissett, J. M. (2008) Maternal and paternal controlling feeding practices: reliability and relationships with BMI, Obes 16, 1552-1558. doi:10.1038/oby.2008.238

23. Hughes, S. O., Power, T. G., O’Connor, T. M., Fisher, J. O., and Chen, T. (2016) Maternal feeding styles and food parenting practices as predictors of longitudinal changes in weight status in Hispanic preschoolers from low-income families, $J$ Obes 2016, 1-9. doi:10.1155/2016/7201082

24. Farrow, C. V., Haycraft, E., and Blissett, J. M. (2015) Teaching our children when to eat: How parental feeding practices inform the development of emotional eating — a longitudinal experimental design, Am J Clin Nutr 101, 908-913. doi:10.3945/ajcn.114.103713

25. Darling, K. E., Fahrenkamp, A. J., Ruzicka, E. B., and Sato, A. F. (2018) Controlling feeding practices mediate the association between food insecurity and parent-reported child BMI percentile, Child Care Health 47, 275-288. doi:10.1080/02739615.2017.1337517

26. Gu, C., Warkentin, S., Mais, L. A., and Carnell, S. (2017) Ethnic differences in parental feeding behaviors in UK parents of preschoolers, Appetite 113, 398-404. doi:10.1016/j.appet.2017.03.001

27. Gregory, J. E., Paxton, S. J., and Brozovic, A. M. (2010) Pressure to eat and restriction are associated with child eating behaviours and maternal concern about child weight, but not child body mass index, in 2- to 4-year-old children, Appetite 54, 550-556. doi:10.1016/j.appet.2010.02.013

28. Dovey, T. M., Staples, P. A., Gibson, E. L., and Halford, J. C. (2008) Food neophobia and 'picky/fussy' eating in children: a review. Appetite 50, 181-193. bttps://doi.org/10.1016/j.appet.2007.09.009

29. Anderson, C. B., Hughes, S. O., Fisher, J. O., and Nicklas, T. A. (2005) Cross-cultural equivalence of feeding beliefs and practices: The psychometric properties of the child feeding questionnaire among Blacks and Hispanics, Prev Med 41, 521-531. doi:10.1016/j.ypmed.2005.01.003

30. Goulding, A. N., Rosenblum, K. L., Miller, A. L., Peterson, K. E., Chen, Y., Kaciroti, N., and Lumeng, J. C. (2014) Associations between maternal depressive symptoms and child feeding practices in a cross-sectional study of low-income mothers and their young children, Int J Behav Nutr Phys Act 11, 75. doi:10.1186/1479-5868-11-75

31. Herpertz-Dahlmann, B., Wille, N., Hölling, H., Vloet, T. D., Ravens-Sieberer, U., and BELLA Study Group. (2008) Disordered eating behaviour and attitudes, associated psychopathology and health-related quality of life: results of the BELLA study, Eur Child Adolesc Psychiatry 17, 82-91. doi:10.1007/s00787-008-1009-9

32. Bauer, K. W., Hearst, M. O., Escoto, K., Berge, J. M., \& Neumark-Sztainer, D. (2012) Parental employment and work-family stress: Associations with family food environments. Social Science \& Medicine, 75(3), 496-504. doi: 10.1016/j.socscimed.2012.03.026.

33. Birch, L., Fisher, J., Grimm-Thomas, K., Markey, C., Sawyer, R., and Johnson, S. (2001) Confirmatory factor analysis of the Child Feeding Questionnaire: A measure of parental attitudes, beliefs and practices about child feeding and obesity proneness, Appetite 36, 201-210. doi:10.1006/appe.2001.0398

34. Jennings, K. M., Loth, K. A., Tate, A. D., Miner, M. H., \& Berge, J. M. (2019) Application of latent profile analysis to define subgroups of parenting styles and food parenting practices. Appetite, 139, 8-18. doi: 10.1016/j.appet.2019.04.001

35. Brown, K. A., Ogden, J., Vögele, C., and Gibson, E. L. (2008) The role of parental control practices in explaining childrens diet and BMI, Appetite 50, 252-259. doi:10.1016/j.appet.2007.07.010

36. Fisher, J., \& Birch, L. (1999) Restricting Access to Foods and Childrens Eating. Appetite, 32(3), 405-419. doi: 10.1006/appe.1999.0231

37. Wertheim, E.H., Mee, V. \& Paxton, S.J. Relationships Among Adolescent Girls' Eating Behaviors and Their Parents' WeightRelated Attitudes and Behaviors. Sex Roles 41, 169-187 (1999) https://doi.org/10.1023/A:1018850111450

38. Cardel, M., Willig, A. L., Dulin-Keita, A., Casazza, K., Beasley, T. M., \& Fernández, J. R. (2012) Parental feeding practices and socioeconomic status are associated with child adiposity in a multi-ethnic sample of children. Appetite, 58(1), 347-353. doi: 10.1016/j.appet.2011.11.005

39. Wehrly, S. E., Bonilla, C., Perez, M., and Liew, J. (2014) Controlling parental feeding practices and child body composition in ethnically and economically diverse preschool children, Appetite 73, 163-171. doi:10.1016/j.appet.2013.11.009

40. Berge, J. M., Tate, A., Trofholz, A., Loth, K., Miner, M., Crow, S., \& Neumark-Sztainer, D. (2018) Examining variability in parent feeding practices within a low-income, racially/ethnically diverse, and immigrant population using ecological momentary assessment. Appetite, 127, 110-118. doi: 10.1016/j.appet.2018.04.006

41. Cachelin, F. M., \& Thompson, D. (2013) Predictors of maternal child-feeding practices in an ethnically diverse sample and the relationship to child obesity. Obesity, 21(8), 1676-1683. doi: 10.1002/oby.20385

42. Caprio, S., Daniels, S. R., Drewnowski, A., Kaufman, F. R., Palinkas, L. A., Rosenbloom, A. L., ... Kirkman, M. S. (2008) Influence of Race, Ethnicity, and Culture on Childhood Obesity: Implications for Prevention and Treatment. Obesity, 16(12), 2566-2577. doi: 10.1038/oby.2008.398 
43. Shea, M., Cachelin, F., Uribe, L., Striegel, R. H., Thompson, D., \& Wilson, G. T. (2012) Cultural Adaptation of a Cognitive Behavior Therapy Guided Self-Help Program for Mexican American Women with Binge Eating Disorders. Journal of Counseling \& Development, 90(3), 308-318. doi: 10.1002/j.1556-6676.2012.00039.x

44. New, C., Xiao, L., and Ma, J. (2013) Acculturation and overweight-related attitudes and behavior among obese Hispanic adults in the United States, Obes 21, 2396-2404. doi: 10.1002/oby.20146

45. Jain, A., Sherman, S. N., Chamberlin, D. L. A., Carter, Y., Powers, S. W., \& Whitaker, R. C. (2001) Why Don’t Low-Income Mothers Worry About Their Preschoolers Being Overweight? Pediatrics, 107(5), 1138-1146. doi: 10.1542/peds.107.5.1138

46. Martinez, S. M., Rhee, K. E., Blanco, E., \& Boutelle, K. (2016) Latino mothers' beliefs about child weight and family health. Public Health Nutrition, 20(6), 1099-1106. doi: 10.1017/s1368980016002962

47. Bruss, M. B., Morris, J. R., Dannison, L. L., Orbe, M. P., Quitugua, J. A., \& Palacios, R. T. (2005) Food, Culture, and Family: Exploring the Coordinated Management of Meaning Regarding Childhood Obesity. Health Communication, 18(2), 155-175. doi: $10.1207 /$ s15327027bc1802_4

48. Blissett, J., Meyer, C., \& Haycraft, E. (2006) Maternal and paternal controlling feeding practices with male and female children. Appetite, 47(2), 212-219. doi: 10.1016/j.appet.2006.04.002

49. Bassett, R., Chapman, G. E., \& Beagan, B. L. (2008) Autonomy and control: The co-construction of adolescent food choice. Appetite, 50(2-3), 325-332. doi:10.1016/j.appet.2007.08.009

50. Chorpita, B. F., Yim, L., Moffitt, C., Umemoto, L. A., and Francis, S. E. (2000) Assessment of symptoms of DSM-IV anxiety and depression in children: A revised child anxiety and depression scale, Behav Res Ther 38, 835-855. doi:10.1016/S00057967(99)00130-8

51. Piqueras, J. A., Martín-Vivar, M., Sandin, B., Luis, C. S., and Pineda, D. (2017) The Revised Child Anxiety and Depression Scale: A systematic review and reliability generalization meta-analysis, J Affect Disord 218, 153-169. doi:10.1016/j.jad.2017.04.022

52. Rosenberg, M. (1965) Rosenberg self-esteem scale (RSE) Acceptance and commitment therapy. Measures package, 61, 52.

53. Ciarroch, J., and Bilich, L. (2006) Acceptance and Commitment Therapy. Measures Package Process measures of potential relevance to ACT(Publication) School of Psychology University of Wollongong.

54. Robins, R. W., Hendin, H. M., \& Trzesniewski, K. H. (2001) Measuring global self-esteem: Construct validation of a singleitem measure and the Rosenberg Self-Esteem Scale, Pers Soc Psychol Bull 27, 151-161. doi:10.1177/0146167201272002

55. Birch, L., Fisher, J., Grimm-Thomas, K., Markey, C., Sawyer, R., and Johnson, S. (2001) Confirmatory factor analysis of the Child Feeding Questionnaire: A measure of parental attitudes, beliefs and practices about child feeding and obesity proneness, Appetite 36, 201-210. doi:10.1006/appe.2001.0398

56. Nowicka, P., Sorjonen, K., Pietrobelli, A., Flodmark, C., and Faith, M. S. (2014) Parental feeding practices and associations with child weight status. Swedish validation of the Child Feeding Questionnaire finds parents of 4-year-olds less restrictive, Appetite 81, 232-241. doi:10.1016/j.appet.2014.06.027

57. Blissett, J., and Haycraft, E. (2008) Are parenting style and controlling feeding practices related?, Appetite 50, $477-485$. doi:10.1016/j.appet.2007.10.003

58. Ballard, R. M., Rodgers, A. B., Khan, L. K., Orleans, C. T., Arkin, E., and Phillips, T. M. (2018) Developing a partnership for change: The National Collaborative on Childhood Obesity Research, Am J Prev Med 54, 465-474. doi:10.1016/j.amepre.2017.12.001

59. Eskilson, A., Wiley, M., Muehlbauer, G., and Dodder, L. (1986) Parental Pressure, Self-Esteem and Adolescent Reported Deviance: Bending the Twig Too Far. ProQuest, 21(83), PMID: 3812058

60. Frederick, C. M., and Grow, V. M. (1996) A mediational model of autonomy, self-esteem, and eating disordered attitudes and behaviors, Psychol Women Q 20, 217-228. doi:10.1111/j.1471-6402.1996.tb00468.x

61. Haycraft, E., Goodwin, H., \& Meyer, C. (2014) Adolescents Level of Eating Psychopathology Is Related to Perceptions of Their Parents Current Feeding Practices. Journal of Adolescent Health, 54(2), 204-208. doi: 10.1016/j.jadohealth.2013.08.007

62. Hautala, L. A., Junnila, J., Helenius, H., Väänänen, A.-M., Liuksila, P.-R., Räihä, H., Välimäki, R., Saarijärvi, S. (2008) Towards understanding gender differences in disordered eating among adolescents. Journal of Clinical Nursing, 17(13), 1803-1813. doi: 10.1111/j.1365-2702.2007.02143.x

63. Grigg, M., Bowman, J., \& Redman, S. (1996) Disordered eating and unhealthy weight reduction practices among adolescent females. Preventive Medicine, 25(6), 748-756. doi: 10.1006/pmed.1996.0115

64. Whyte, H., Findlay, S. M., \& Canadian Paediatric Society, Adolescent Health Committee. (2004) Dieting in adolescence, Paediatrics \& Child Health, 9, 487-491, https:// doi.org/10.1093/pch/9.7.487

65. Nowicka, P., Flodmark, C. E., Hales, D., and Faith, M. S. (2014) Assessment of parental overt and covert control of child's food intake: A population-based validation study with mothers of preschoolers, Eat Behav 15, 673-678. doi:10.1016/j.eatbeh.2014.10.001

66. O'Connor, T. M., Mâsse, L. C., Tu, A. W., Watts, A. W., Hughes, S. O., Beauchamp, M. R., Baranowski, T., Pham, T., Berge, J. M., Fiese, B., Golley, R., Hingle, M., Kremers, S. P. J., Rhee, K. E., Skouteris, H., \& Vaughn, A. (2017) Food parenting 
practices for 5 to 12 year old children: a concept map analysis of parenting and nutrition experts input. International Journal of Behavioral Nutrition and Physical Activity, 14, 122. https://psycnet.apa.org/ record/2017-40559-001

\section{ABOUT STUDENT AUTHOR}

Paulina Mozdzierz will graduate in May 2021 with a bachelor's degree in Health Promotion and Disease Prevention and a minor in Business Economics. She plans on enrolling in a physician assistant program after graduating.

\section{PRESS SUMMARY}

Mothers may attempt to control their children's food intake in order to make the child gain or lose weight. The purpose of this study was to determine if these practices have an association with weight and mental health of children. Results were based on 202 pairs of mothers and their children. The following findings were determined as part of the results: mothers pressuring their children to eat was related to a lower child body mass, restricting children from eating certain foods was related to a higher child body mass, and mothers pressuring their children to eat was related to lower child self-esteem. 